\title{
Directional Interfaces for Wearable Augmented Reality
}

\author{
Volkert Buchmann \\ HIT Lab NZ \\ University of Canterbury \\ Christchurch, New Zealand \\ volkert.buchmann@ \\ canterbury.ac.nz
}

\author{
Mark Billinghurst \\ HIT Lab NZ \\ University of Canterbury \\ Christchurch, New Zealand \\ mark.billinghurst@ \\ canterbury.ac.nz
}

\author{
Andy Cockburn \\ Department of Computer \\ Science \\ University of Canterbury \\ Christchurch, New Zealand \\ andy@cosc.canterbury.ac.nz
}

\begin{abstract}
Wearable Augmented Reality can be used to overlay information onto the real world. Directional interfaces in wearable Augmented Reality aid users to orient themselves so that previously invisible targets are now inside their field of view. This is relevant when the user tries to find the next waypoint during a navigational task. We surveyed directional interfaces that have been used in Augmented Reality previously and compared their efficiency. We have found that a circular compass is the most efficient way to provide orientation cues.
\end{abstract}

\section{Keywords}

Wearable Augmented Reality, Navigation, Directional Interfaces, Haptics

\section{INTRODUCTION}

Wearable Augmented Reality (AR) interfaces are relatively new systems that allow users to see virtual graphics superimposed over the real world in real time. They can be used for applications such as architectural visualization [18], gaming [3], or as an aid for navigation [9]. In our research we are particularly interested in using wearable AR systems for improving outdoor surveying.

Being able to locate the survey points is an important part of surveying. This paper compares different ways that a wearable AR interface can be utilised to help the user to orient themselves in a certain direction. We have researched these interfaces in the context of navigation where the user moves from waypoint to waypoint. Once a waypoint is reached, the user needs to orient themselves towards the next waypoint. We assume that these waypoints are very easy to recognise once they are in the user's field of view, as they will be highlighted by the AR system. However, when they are outside the user's field of view, an interface is needed to help the user face the correct direction.

Permission to make digital or hard copies of all or part of this work for personal or classroom use is granted without fee provided that copies are not made or distributed for profit or commercial advantage, and that copies bear this notice and the full citation on the first page. To copy otherwise, to republish, to post on servers or to redistribute to lists, requires prior specific permission and/or a fee. CHINZ 2008, July 2, 2008, Wellington, New Zealand (C) 2008 ACM 978-1-60558-467-6/07/0008...\$5.00
This need stems mainly from the very limited field of view that AR head mounted displays (HMDs) provide. For example, the camera of our video see-through HMD only provides a $50^{\circ}$ horizontal field of view, significantly smaller than the typical human field of view of $180^{\circ}$ degrees. This small virtual window into the real world causes a loss of context and makes it difficult for the user to see the next virtual waypoint as they walk towards the current one.

While navigation in AR itself has been the subject of previous work, interfaces for direction finding have mostly only been mentioned in passing and formal comparisons between them are the exception. Previously, [1] have formally compared a limited selection of visual and audible directional cues. In this paper, we survey directional interfaces previously used in wearable AR systems and formally compare several of them. The interfaces include visual as well as audible and haptic cues, and the most efficient one is a modified circular compass.

In the next section, we will provide a survey of previous work on directional interfaces in wearable AR. After this, we will describe our implementations of these interfaces and compare them in a formal study.

\section{BACKGROUND}

A variety of directional interfaces have previously been described for wearable AR. They can be classified into three categories:

- no angular information

- indirect angular information

- direct angular information

An example of an interface that provides no angular information are left and right arrows that only indicate if the user needs to turn clockwise or counter-clockwise [16]. An interface that provides indirect angular information is a compass that informs the user how far they have to turn [5]. Direct angular information can be provided by a haptic belt that taps the user from the correct real world direction without the need for the user to map from a visual representation to the real world [17]. While it is easy to assume that the interfaces would be more efficient the more direct they are and the more angular information they provide, this is not necessarily true, as our experiment will show. 
Most directional interfaces are head-stabilised, meaning that they always appear on the same position on the screen, independent of the user's head or body movements. We will distinguish between two types of head stabilised interfaces: head-up displays (HUDs), which are two-dimensional interfaces drawn directly on the screen (see for example figure 1(a)), and perspective interfaces, which are presented to the user at an angle (see for example figure 1(b)). Normal AR objects are world-stabilised, meaning that they are correctly registered in 3D and appear as part of the real environment. A head-stabilised interface has the advantage that it is always visible, independent of head orientation, while a worldstabilised interface has the advantage of directly showing a location in the real world.

Left/right arrows are the simplest directional visualisation (see figure $1(\mathrm{~d})$ ). These arrows are a HUD and indicate whether the user has to rotate left or right $[1,16,13,14]$. No angular information is provided.

Turning signals are arrows that indicate whether the user has to turn left, right, go forward or turn around $180^{\circ}[9$, 8]. Left and right turns are traditionally visualised as bent arrows. As opposed to left/right arrows, turning signals are more appropriate for navigating in a grid-like environment with $90^{\circ}$ turns such as buildings or streets, while we are interested in interfaces for arbitrary directions.

Maps have been used to display the user's position and orientation. The orientation of the map on screen is a relevant factor in this case. A forward-up $[14,7]$ map is automatically rotated, so that the user's direction is aligned with the "up" direction of the map. A north-up map [19] is aligned with North and is not rotated. Darken et al. found that forward-up maps are more efficient for simple target finding, while north-up maps are more efficient for tasks that require context [4]. Maps are usually head-stabilised although there have also been hand-stabilized $[7,11]$ implementations. Some of the systems use a HUD map, while others use a perspective map. All of these map types provide indirect angular information. Suomela et al. compared HUD and perspective maps and found that HUD maps were more efficient [14]. However, they hypothesized that this was due to the low resolution of their display. Some of the maps used are two-dimensional [19], while others followed the World-InMiniature metaphor and used three-dimensional maps [11].

We did not use a map interface in our evaluation, as they are more concerned with providing contextual rather than directional information. We assume that the wealth of information presented by such an interface would not make it efficient for our purposes. If we were to reduce the amount of information displayed on the map to a minimum - the user's current location and the location of the next target we would have an interface too similar to the compass to be included in the experiment.

A compass has been used in several wearable AR applications [5, 11, 8] and models a familiar tool (see figure 1(a)). In the context of this paper, a compass is forward-up and the needle does not point to North, but to the target. It provides indirect angular information.
A horizontal compass maps a part or the whole range of a compass onto a horizontal line with the centre of the line being aligned with the user's line of sight (see figure 1(c)) $[16,13]$. The compass needle moves horizontally along the compass and provides indirect angular information.

Audio cues can be distinguished into spatial audio that simulates a sound beacon at the location of the target [1] and speech interfaces with pre-recorded [12] or synthesized [10] speech. [1] used a continuous white noise loop as their audio beacon. [10] used an audio beacon as well as synthesized speech to inform severely visually impaired users about the heading of the target in degrees or clock face positions. The beacons were played back every $700 \mathrm{~ms}$ while the speech output was played back every $1700 \mathrm{~ms}$.

Haptic cues have the advantage of not taking up screen real estate while not being susceptible to environment noise as sound cues are [10]. [10] describe a turning signals interface based on the "rabbit" display [15]. The interface taps the user's shoulders to indicate left and right turns. It taps the user's neck to indicate that the target is straight ahead. For each case, double taps are made every 700ms. After user testing, [10] suggest that the tapping frequency for the neck should be reduced. [17] have tested a belt with eight vibrators and have found that their participants were able to use it for waypoint navigation. [20] have proposed a haptic belt with four haptic actuators to indicate forward, backward, left and right turning signals.

There have not yet been a comprehensive description or comparison of these different directional interfaces, and most authors do not say how well they performed. Notable differences are the studies by [1] and [10]. [1] compared the efficiency of HUD left/right arrows and an audio beacon for a rotation task. Both these two cues alone as well as their combination performed faster than no cue. There was no difference between the performance of either cue or their combination. [10] compared three directional interfaces for users with severe visual impairments: a haptic interface and two audio interfaces. The haptic interface taps the user on the shoulder for changes in direction, and taps the neck for forward movements. This is compared to a spatial audio beacon and a speech interface that informs the user every 1.7 seconds about how far off course the target is, either in degrees or clock face positions. All three interfaces were compared for their performance for crossing the road. Users preferred the haptic interface which also performed best. The audio interfaces did not work well in noisy environments.

Haptic belts have not yet been compared to other directional interfaces, but there has been a study evaluating the feasibility of such a device. The study found that participants were able to use the belt to follow a complex path without any visual or acoustic help [17].

As can be seen, developers of wearable AR systems have found it necessary to integrate directional interfaces into their systems. A wide range of possible interfaces have been used with little or no assessment of their efficiency. Unlike previous work, in this paper we both describe several different modalities of interfaces for providing directional cues 
and a rigorous user study evaluating these interfaces.

In the next section, we will describe the interfaces we have developed and then the comparative evaluation we conducted.

\section{IMPLEMENTED DIRECTIONAL INTER- FACES}

We implemented six directional interfaces based on the most promising techniques described in the research literature. We developed four visual interfaces based on a circular compass, a horizontal compass and left/right arrows (see figure 1). We also developed a haptic belt and an interface that uses a spatial audio beacon.

\subsection{General Design Decisions}

During the implementation of the interfaces, we had to make design decisions that we applied to most of our interfaces.

We were concerned whether head roll (leaning the head to the side) would make our head-stabilised visualisations confusing. As long as the user holds the head perpendicular to the ground, the virtual imagery would align well with the real world. However, if the head was tilted, an interface supposed to point to the side would now also point up or down. We tried to correct for this by making the visualisations world-stabilised with respect to camera roll but head-stabilised with respect to the other 5 degrees of freedom. Now they would always appear level with respect to the real world. We found this to be more confusing than helpful. Directional interfaces with world-stabilised roll appeared to be tilted rather than balanced horizontally. This became more apparent the more the head was tilted. Thus, our visualisations are fully head-stabilised.

\subsection{Left/Right Arrows}

We have implemented a simple interface with left/right arrows as shown in figure $1(\mathrm{~d})$. In order to avoid rapid jumping between the arrows for targets directly behind the user, we used a dynamic threshold depending on which arrow is currently displayed.

\subsection{Circular Compasses}

We have implemented two versions of the circular compass: a HUD version (figure 1(a)) and a perspective head-stabilised version (figure $1(\mathrm{~b})$ ). Both are based on the same drawing algorithm. The square as seen in figures $1(\mathrm{a})$ and $1(\mathrm{~b})$ is for our participants to easily distinguish between the two compass visualisations.

We will call the part of the human field of view that is not covered by the HMD the phantom field of view. When using the system, a target placed in the phantom FOV would lead to conflicting information, especially when the target was close to the edge of the HMD's FOV. In early trials with the circular compass interface, users sometimes perceived the compass needle to point to an object "in front of them" wile the target was not visible in the HMD. This occurs because the user assumes that the camera image covers a larger part of their natural FOV. Some users were not able to get accustomed to the differences in field of view, and even after repeated use of the system the compass visualisation remained confusing to them. To address this problem, we explicitly incorporated the two different field of views in the compass visualisation (see figure 1(a)): the camera's field of view is highlighted as an arc in the compass, while the human field of view is highlighted as compass markers at $90^{\circ}$ and $-90^{\circ}$. In addition to this, we placed a compass marker at 180 degrees. I pilot testing, this visualisation eliminated the confusion between the camera and human fields of view.

\subsection{Horizontal Compass}

Our horizontal compass (figure 1(c)) is inspired by the context compass [13]. However, while the context compass visualises targets within the user's FOV, our horizontal compass visualises targets that the user cannot see. It works similar to a rear-view mirror, with the needle moving left as the user turns left. We have included this visualisation since most people are used to rear-view mirrors. A horizontal visualisation might also be more easily integrated into a HMD interface than a circular compass.

Our horizontal compass covers all directions that are not within the user's FOV. It has the same $90^{\circ},-90^{\circ}$ and $180^{\circ}$ markings as the circular compass. In figure $1(\mathrm{c})$, a target is indicated at $-120^{\circ}$, requiring the user to turn $120^{\circ}$ to the left. Since the compass needle is drawn right of the $-90^{\circ}$ marker, the user can see that the target is behind him. As the user turns left, the needle moves to the left as well, just as in a mirror. As the needle disappears left off the screen, the target appears in the user's FOV and moves from left to right.

\subsection{Audio Beacon}

We have implemented a spatial audio beacon to indicate the position of a target. Descriptive cues such as the one described by [10] will probably be slower due to the duration of the spoken instructions, and thus we have decided not to use it. Sound was played back with the OpenAL library, using DirectSound3D as the underlying sound system. A generic sound card and headphones were used. The audio beacon was a continuous white noise loop similar to the one used by [1]. The beacon was head-stabilised except for yaw, which was world-stabilised.

\subsection{Haptic Belt}

Based on the positive results reported in the literature, we decided to include a haptic belt in the evaluation. We decided to use six actuators to compromise between cost and efficiency. The actuators were distributed around the user's waist as shown in figure 2(b). The chosen configuration spreads the actuators evenly around the user's waist with the exception of the front. Note that the irregular shape of the human body means that an even distribution on the body's surface results in uneven angular distribution of the actuators. In figure 2(b), the straight lines through the centre of the body indicate $45^{\circ}$ angles, showing that there is a $90^{\circ}$ distance between the two back and between the front actuators, while they are evenly spaced across the body's surface. For directions that fall between two actuators, we interpolate and trigger both actuators.

For the actuators, we initially used small buzzers of the same type used for our fingARtip system [2]. However, we found that some people were not able to feel the buzzers when 


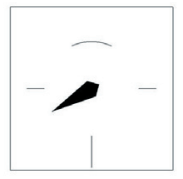

(a) The HUD compass

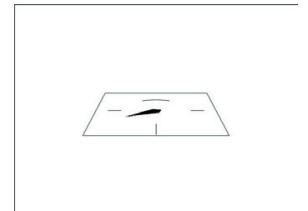

(b) The perspective compass

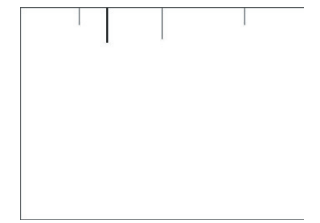

(c) The horizontal (d) Left/right arrows
compass

Figure 1: Our four directional visualisations. All are indicating a target at $-120^{\circ}$

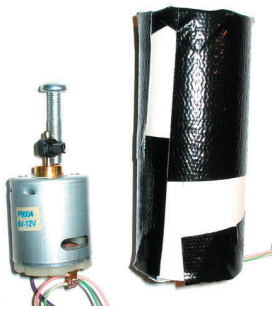

(a) The belt's haptic actuator with and without the plastic casing.

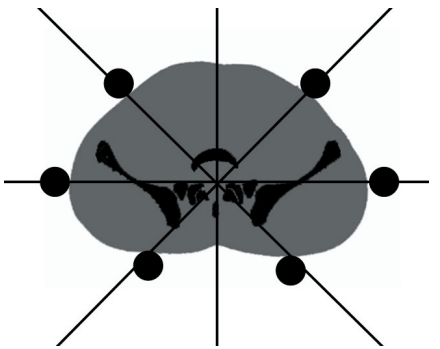

(b) A cross-section of a waist showing the hip bone and the configuration of the haptic belt's actuators. The front of the waist points to the top, and the lines indicate $45^{\circ}$ steps.

Figure 2: The haptic belt.

placed on soft tissue areas while the vibrations were felt strongly when the actuators were placed close to a bone. See figure 2(b) for an indication of the bone distribution in the waist area. As a result, we decided to use a tapping signal rather than vibration. We used motors with eccentric weights (see figure $2(\mathrm{a})$ ), as they are used for vibration actuators. The motors were only switched on for short periods of time, just long enough for one revolution, so that they produced successive tapping motions rather than vibrations. The actuators were activated for $100 \mathrm{~ms}$ and then switched off for $67 \mathrm{~ms}$.

The orientation of the belt was tracked independently of the HMD, and the belt would only signal the target's direction if the target was not in the HMD's FOV.

\section{EXPERIMENT}

The aim of this experiment is to compare the performance of the selected directional interfaces in an AR environment. We asked the participants to orient themselves while standing on the spot using the different directional interfaces and measured task completion time and accuracy.

The experiment follows a repeated measures factorial design with seven different interface conditions: no help, left/right arrows,HUD compass, perspective compass, horizontal compass, spatial sound, haptic belt.

The dependent measures were task completion time and the number of times the user overshoots the target direction.
Participants were also asked to answer selected questions from the NASA TLX questionnaire [6].

\subsection{Procedure}

Each participant completed seven direction finding tasks for each interface type. The target directions were symmetrical with respect to the user's left and right hand side to eliminate bias. Targets were placed at $50^{\circ}, 90^{\circ}, 130^{\circ}, 180^{\circ}$ and at $-50^{\circ},-90^{\circ}$ and $-130^{\circ}$. Considering that the HMD's FOV is $50^{\circ}$, the participants only had to turn $25^{\circ}, 65^{\circ}, 105^{\circ}$ and $155^{\circ}$, respectively. The order of the target directions between the interfaces and the order of the interfaces between participants were each counter-balanced using a Latin square. Experiments took on average of 30 minutes, including completion of the questionnaires.

\subsection{Apparatus}

Participants wore a monoscopic $800 * 600$ video see-through HMD. Target directions were displayed as vertical red lines. Participants were asked to roughly orient themselves towards the same start location before each task. The target locations were then placed relative to the participant's actual orientation to ensure comparable measurements. For each task, the direction of the target was displayed using the current interface type. The participants were then asked to orient themselves as quickly and accurately as possible towards the target. A task trial ended when the target was in the participant's field of view for one second. This second was then subtracted from the measured task completion time. See figure 3 for a picture of the experiment setup. 


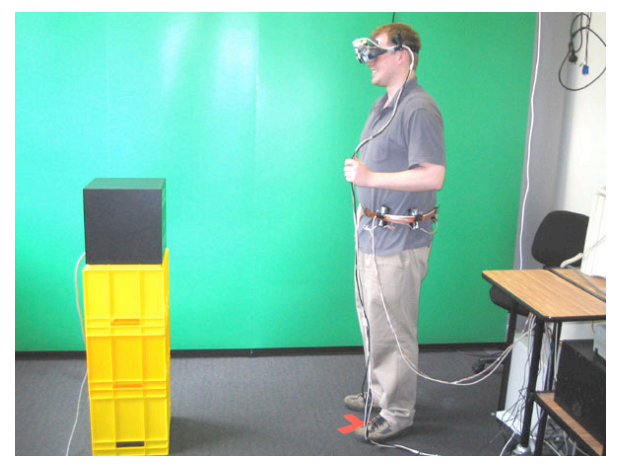

Figure 3: The experiment setup for the haptic belt condition. Participants wore a video see-through HMD and were tracked with a Flock of Birds magnetic tracker.

The user's head orientation and the haptic belt were tracked with a Flock of Birds magnetic tracker with a resolution of $0.1^{\circ}$ and an accuracy of $0.5^{\circ}$. Only yaw information was used to overlay AR content onto the HMD.

\subsection{Participants}

We had 14 participants ranging from 20 to 45 years, all students at the University of Canterbury. 11 were male, 3 were female. 3 of them had used VR systems before, and none of them had experienced AR before.

\subsection{Results}

The two circular compasses performed best of all the interfaces. They were faster, resulted in fewer overshootings and were preferred by the participants.

\subsubsection{Task Completion Time}

The fastest interface was the HUD compass, closely followed by the perspective compass. As expected, the "no help" condition showed the slowest performance. An analysis of variance shows a significant difference between the interfaces $(F(6,90)=10.44, p<0.001)$. Figure $4($ a) shows the average turning speed for each interface in milliseconds per degree.

The average task completion times split up by target distance and interface are plotted in figure $4(\mathrm{~d})$. We can see that the two compass interfaces both performed about a second faster for each target direction than the "no help" condition. Linear regression can show for 4 of the interfaces that they have linear performance, as shown in table 1.

\subsubsection{Overshooting}

The results were even more dramatic for overshooting. Again the two circular compasses performed best, and there was a significant difference between the interfaces $(F(6,90)=$ $5.11, p<0.001$ ) (see figure $4(\mathrm{~b})$ ). These results mean that a user would overshoot once in 100 tasks when using the HUD compass compared to 27 times in 100 when using left/right arrows.

While overshooting is not a problem for target acquisition using a mouse or a similar interface, it is desirable to minimize unnecessary head movement. To stay focused and avoid disorientation - especially with a significantly reduced field of view - the AR system needs to guide the user in such a way as to avoid unnecessary head movement. The two circular compasses achieve near-perfect performance for this.

As opposed to the two circular compasses, there was high variance across the other interfaces.

\subsubsection{Subjective Measures}

The subjective measures generally agreed with the objective results that we have just described. We asked our participants to rank the interfaces from 1 ("liked best") to 7 ("liked least"). A Friedman test showed that there was a significant difference between the interfaces $\left(\chi_{r}^{2}=47.18, d f=6, N=\right.$ $13, p<0.001)$, again, with the two circular compasses performing best (see figure $4(\mathrm{c})$ ).

We asked the participants four questions for each interface which they answered on a Likert scale from 1 ("disagree") to 7 ("agree"). Friedman tests showed that there was a significant difference between the interfaces for each of the questions, with the HUD compass always rated best and the perspective compass always rated second best. The questions were "I performed well" $\left(\chi_{r}^{2}=39.24, d f=6, N=13, p<\right.$ $0.0001)$, "I found the task easy to complete with this interface." $\left(\chi_{r}^{2}=40.45, d f=6, N=12, p<0.0001\right)$, "It was always easy to understand and follow the directions from the interface." $\left(\chi_{r}^{2}=32.74, d f=6, N=12, p<0.0001\right)$ and "I felt comfortable using the interface." $\left(\chi_{r}^{2}=26.49, d f=\right.$ $6, N=12, p<0.0001)$.

The comments participants made also agreed with the statistical data. Several users commented on the sudden ease of direction finding when switching to one of the circular compasses for the first time, saying "oh, this is much better" and so on. The circular compasses received overwhelmingly positive comments such as "[it] tells you exactly where to turn before you turn", "[it was] very easy to follow", "perfect" and so on. The markings on the circular compasses were also well received with several comments such as "the FOV [marking] was very helpful and stopped me from overshooting" and "having the $90^{\circ}$ and $180^{\circ}$ markers were helpful". Some participants also highlighted issues with the perspective compass as compared to the HUD version with comments such as "it was distorted a bit, so I found it harder to tell the exact 


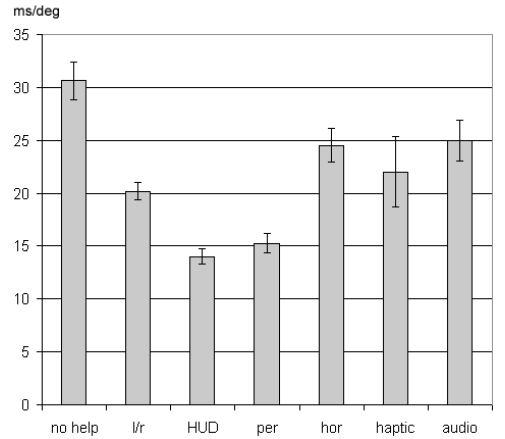

(a) Normalized means for milliseconds per degree for each interface.

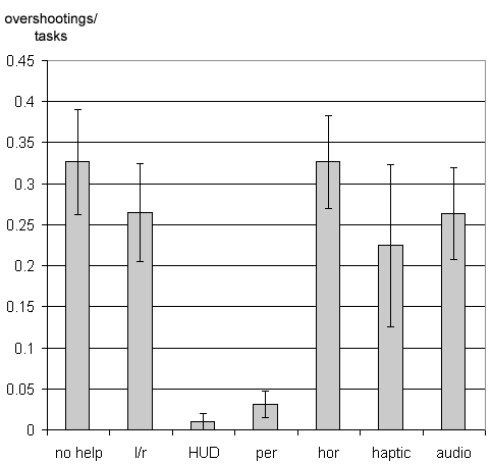

(b) Average number of overshootings per task for each interface.

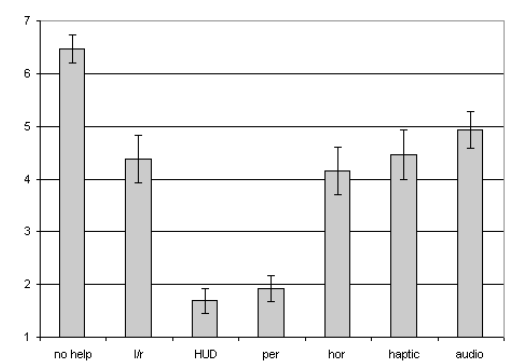

(c) Ranking from 1 "liked best" to 7 "liked least".

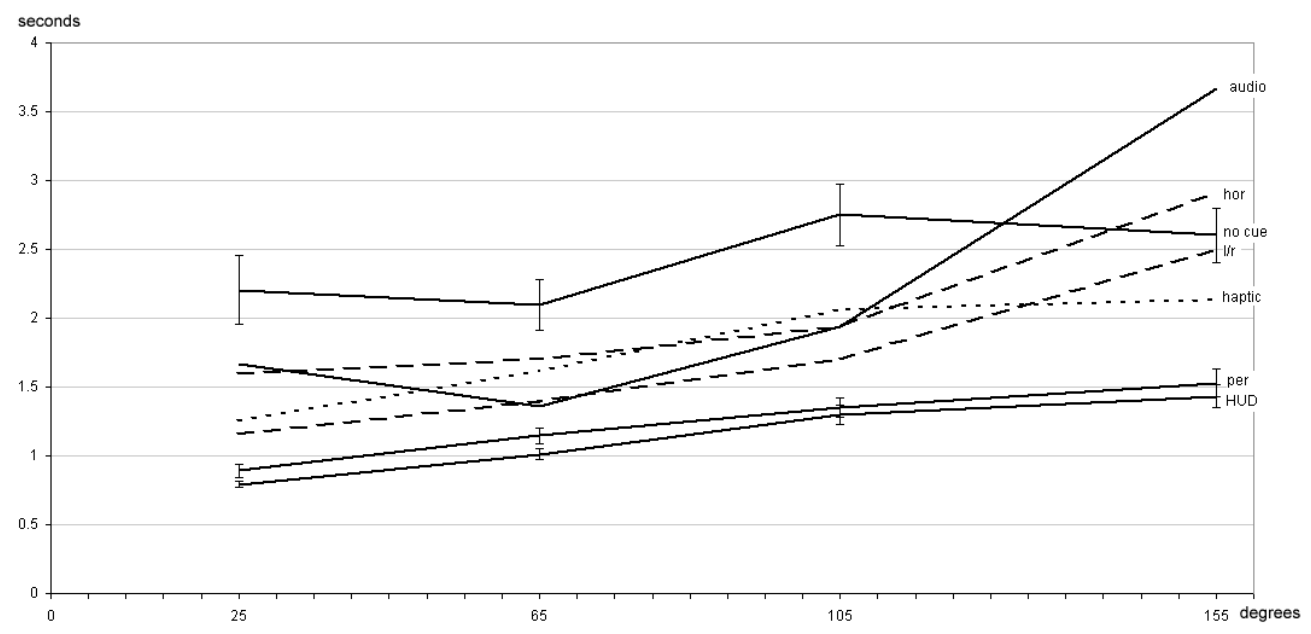

(d) A time/degree diagram for the interfaces. Only some interfaces have error bars to avoid clutter.

Figure 4: The quantitative measures

point I was turning to" and "had to 'read' the perspective a little more". One participant noted that the HUD compass took up a lot of screen real estate as compared to the other interfaces.

The other visual interfaces did not receive such positive comments, with several participants noting that with the left/right arrows, they "didn't know how far to turn". The horizontal compass was called "confusing" and "troublesome" by most participants, with some noting that they "might get better with practice" or that it was "good practice for [their] brain". One of the participants strongly preferred the horizontal compass and even ranked it the highest. Coincidentally, he was the second slowest participant with this interface.

The non-visual cues also received mixed comments. Spatial audio was not perceived as an efficient interface, with several stating that "it was hard to tell where exactly the noise was coming from" and others making comments such as "confusing when target is behind". Several participants also noted that they did not like the audio sample or that this interface would interfere with conversations or listening to music. However, one of the participants noted that this was the least obtrusive interface.

Opinions on the haptic belt were more divided. While some found the interface "fun" and "easy to follow", several participants said that they felt "uncomfortable" or "slightly uncomfortable". Others noted the problems that we encountered while building the belt: "some parts of the body feel [the] motor [more strongly] than others. So [the] signal is stronger in different directions." A few participants commented on the low resolution of the device: "it didn't tell me exactly where the target was, it only guided me in the general direction." and "ambiguous vibrations when the target is close".

\section{DISCUSSION}

The two circular compasses are the obvious winners of this formal comparison. They have not only been shown to perform significantly better than most of the other interfaces, but they have also been rated best by the participants. The results from these conditions also have relatively small variance, showing that most participants were equally able to use them. 
Table 1: Regression Analysis results

\begin{tabular}{|c|c|c|c|c|}
\hline interface & $R^{2}$ & $p$ & $\mathrm{a}$ & $\mathrm{b}$ \\
\hline HUD compass & 0.97 & $<0.05$ & 5.08 & 690.00 \\
\hline perspective compass & 0.98 & $<0.01$ & 4.88 & 802.85 \\
\hline left/right arrows & 0.94 & $<0.05$ & 10.17 & 798.41 \\
\hline haptic & 0.91 & $<0.05$ & 7.03 & 1150.87 \\
\hline
\end{tabular}

\subsection{Haptic Belt}

It is notable that the haptic belt showed relatively large variance in its performance. We believe that there are four possible main reasons for this. Firstly, the belt had by far the lowest resolution of the interfaces that provided angular information. Compared to the belt's six actuators, the horizontal compass, for example, had a 133 times better resolution. Considering its low resolution, the belt performed surprisingly well. Secondly, the haptic actuators had to be adjusted for each participant, so that they were positioned at the same angular direction. The largest participant needed a belt $20 \mathrm{~cm}$ longer than the thinnest participant did. We positioned the actuators according to the diagram shown in figure 2(b), but did not perform accurate calibration. We believed that this better reflects how the belt would be used in practice. In hindsight, accurate calibration would have been preferable. Another possible contributing factor was the relative inexperience of the participants with such a device. Most people will not have much practice in quickly orienting themselves towards the direction of a haptic signal. Thus, the perceived relation between their waist and the real world coordinates might be distorted. This is a factor that can be reduced by training. Another possible factor is the resolution of haptic sensors in the waist area. It is possible that we cannot physically differentiate well enough between the signals, making accurate perception of the signal impossible. This is especially true for vibration, which can be felt over a larger area than other stimuli such as taps. While we tried to drive the actuators so that hey tap rather than vibrate, vibration could still be felt, which may have made perception of the stimuli less precise. Unfortunately, although miniature vibrators are readily available - such as the ones used in cell phones - we have not found off-the-shelf tapping actuators.

We believe that the performance with the haptic belt could be improved by making the above modifications. However, to be a practical solution, the device will need an accurate calibration procedure that is not prohibitive in everyday use. Based on our results and the costs incurred by the additional tracking and calibration requirements, we conclude that the belt is not an alternative to visual directional interfaces. However, we were able to confirm previous findings that haptic belts do work, and we believe that the may be helpful to blind users or in situations where HMDs cannot be used.

\subsection{Audio Beacon}

As shown in figure $4(\mathrm{~d})$, the audio beacon was most efficient for $90^{\circ}$ and $-90^{\circ}$ turns, which was to be expected. In these locations, there is the least ambiguity in audio beacons. There was a sharp rise in task completion time for the audio beacon placed at $180^{\circ}$, making this condition the worst for this target. An audio beacon at this location sounds exactly the same for each ear, apparently causing confusion for our participants. We noticed during the experiment that most of the participants initially did not turn in either direction when this stimulus was provided. Instead, they tried to find out which side the sound was coming from before they started to turn. Since the sound provided to each ear was near identical, this condition proved to be somewhat like a deadlock.

It should also be noted that the data collected for the $180^{\circ}$ direction may not be as reliable as for the other directions, as the sample size here is only half the size as for the other directions. This is because this direction was not mirrored as the other ones.

\subsection{Horizontal Compass}

While the horizontal compass performed slightly worse than most other interfaces, we believe that its performance may be greatly improved. Our research focused on visualizing targets outside the user's field of view, and thus we only concerned ourselves with the portion of the horizontal compass that was outside the user's field of view, creating an interface that works similar to a rear-view mirror. While it was straightforward to explain the other interfaces to our participants, the horizontal compass was difficult to explain and tricky to master. We now believe that a more traditional horizontal compass such as the one originally described by [13] would be more efficient. The scale could also be extended to cover all 360 degrees. Further research will be necessary to compare the performance of this interface to that of the circular compasses.

\subsection{Future Work}

As already pointed out, we would like to revisit the horizontal compass.

Another important question is how directional interfaces can be integrated in wearable outdoor AR systems. The interface that showed the best performance in our experiment occupied a rather large and obtrusive area in the centre of the screen. This is not ideal for regular use. We will have to find out how small the compass can be made, if it has to be centered horizontally, and if it should be on the screen all the time or only when the target is not in view. If the revised horizontal compass performs comparably, then we will investigate its performance for everyday use, as its shape allows for better integration into screen-based interfaces.

While this question is outside the scope of our work, it would also be important to investigate how a directional interface can be integrated with a contextual interface such as a map. This would allow us to explore which interface would allow 
users to understand the direction of more than one target, and how many targets each interface could support.

\section{CONCLUSION}

We have surveyed directional interfaces for wearable Augmented Reality and found a variety of directional cues in the research literature. No comprehensive comparative study had been undertaken before.

We have developed and formally compared six directional interfaces for target searching in AR, and we have found that a solution using a circular compass is significantly better than the other interfaces. We have found that it is necessary to add a visualisation of the user's field of view to the compass to make it an efficient cue.

We have developed and tested a haptic belt as a directional interface. While the belt did not perform as well as the circular compass, it performed as well as the other interfaces, and we believe it may be a viable option for systems that require non-visual interfaces, such as systems for the blind.

In conclusion, this paper offers a comprehensive formal comparison of a variety of directional interfaces. Developers of wearable AR systems will be able to use this study as a guideline when choosing an interface that suits their need.

\section{REFERENCES}

[1] M. Billinghurst, J. Bowskill, N. Dyer, and J. Morphett. An evaluation of wearable information spaces. In VRAIS '98: Proceedings of the Virtual Reality Annual International Symposium, page 20, Washington, DC, USA, 1998. IEEE Computer Society.

[2] V. Buchmann, S. Violich, M. Billinghurst, and A. Cockburn. Fingartips: gesture based direct manipulation in augmented reality. In Proceedings of the 2nd international conference on Computer graphics and interactive techniques in Australasia and South East Asia, pages 212-221. ACM Press, 2004.

[3] A. D. Cheok, F. S. Wan, K. H. Goh, X. Yang, W. Liu, F. Farzbiz, and Y. Li. Human pacman: A mobile entertainment system with ubiquitous computing and tangible interaction over a wide outdoor area. In Mobile HCI 2003, pages 209-223, 2003.

[4] R. P. Darken and H. Cevik. Map usage in virtual environments: Orientation issues. In VR '99: Proceedings of the IEEE Virtual Reality, pages 133-140, Washington, DC, USA, 1999. IEEE Computer Society.

[5] S. Feiner, B. MacIntyre, T. Höllerer, and A. Webster. A touring machine: Prototyping $3 \mathrm{~d}$ mobile augmented reality systems for exploring the urban environment. In Proceedings of ISWC 1997, pages 74-81, 1997.

[6] S. Hart and L. Staveland. Development of nasa-tlx (task load index): Results of empirical and theoretical research. In P. a M Hancock, editor, Human Mental Workload, pages 139-183, 1988.

[7] T. Höllerer, S. Feiner, T. Terauchi, G. Rashid, and D. Hallaway. Exploring mars: Developing indoor and outdoor user interfaces to a mobile augmented reality system. Computers and Graphics, 23(6):779-785, 1999.

[8] J. Newman, D. Ingram, and A. Hopper. Augmented reality in a wide area sentient environment. In $I S A R$ '01: Proceedings of the IEEE and ACM International Symposium on Augmented Reality (ISAR'01), page 77, Washington, DC, USA, 2001. IEEE Computer Society.

[9] G. Reitmayr and D. Schmalstieg. Location based applications for mobile augmented reality. In CRPITS '03: Proceedings of the Fourth Australian user interface conference on User interfaces 2003, pages 65-73, Darlinghurst, Australia, Australia, 2003. Australian Computer Society, Inc.

[10] D. Ross and B. Blasch. Evaluation of orientation interfaces for wearable computers. In The Fourth International Symposium on Wearable Computers, pages 51-58, 2000.

[11] D. Schmalstieg and G. Reitmayr. The world as a user interface: Augmented reality for ubiquitous computing. In Central European Multimedia and Virtual Reality Conference 2005 (CEMVRC 2005), 2005.

[12] T. Sephton. Teaching agents for wearable augmented reality. In Proceedings of ISWC 2003, 2003.

[13] R. Suomela and J. Lehikoinen. Context compass. In The Fourth International Symposium on Wearable Computers, pages 147-154, 2000.

[14] R. Suomela, K. Roimela, and J. Lehikoinen. The evolution of perspective view in walkmap. Personal Ubiquitous Comput., 7(5):249-262, 2003.

[15] H. Z. Tan and A. Pentland. Tactual displays for wearable computing. In First International Symposium on Wearable Computers, 1997. Digest of Papers, pages 84-89, 1997.

[16] B. Thomas, V. Demczuk, W. Piekarski, D. Hepworth, and B. Gunther. A wearable computer system with augmented reality to support terrestrial navigation. In 2nd International Symposium on Wearable Computers, pages 168-171, 1998.

[17] J. VanErp, H. VanVeen, and C. Jansen. Waypoint navigation with a vibrotactile waist belt. $A C M$ Transactions on Applied Perception, 2(2):106-117, 2005.

[18] V. Vlahakis, J. Karigiannis, M. Tsotros, M. Gounaris, L. Almeida, D. Stricker, T. Gleue, I. T. Christou, R. Carlucci, and N. Ioannidis. Archeoguide: first results of an augmented reality, mobile computing system in cultural heritage sites. In Proceedings of the 2001 conference on Virtual reality, archeology, and cultural heritage, pages 131-140. ACM Press, 2001.

[19] V. Vlahakis, J. Karigiannis, M. Tsotros, N. Ioannidis, and D. Stricker. Personalized augmented reality touring of archaeological sites with wearable and mobile computers. In Proceedings of ISWC 2002, pages $15-22,2002$.

[20] J. S. Zelek and M. Holbein. Feeling where you are wearable haptic directional belt: a device for wayfinding and orientation for people who are blind. In $9 e$ édition du Concours Innovation Recherche 2006 , 2006. 